\title{
Brucella melitensis: A Rarely Suspected Cause of Infections of Genitalia and the Lower Urinary Tract
}

\author{
Stamatiou K., Polyzois K., Dahanis S. Lambou T. and Skolarikos A. \\ General Hospital of Elefsina “Thriassio", Urology Department; Elefsina, Greece
}

\begin{abstract}
We examined the clinical presentation and outcome of Brucellar infections of genitalia and the lower urinary tract through a review of the medical records of 10 cases of male patients with brucellar infections of the genitalia and lower urinary tract. The mean age of the patients with brucellosis was 49.2, (median 52, range 15-77 years). Eleven out of 17 patients were rural residents, 15 reported that they might have consumed unpasteurized dairy products) and four reported occupational exposure. Symptoms onset was acute in almost all cases. Scrotal pain, epidedimal swelling and fever were the most common symptoms. The Wright test was positive in 13 patients, while Brucella sp. was isolated from blood cultures in six cases. Only two patients were found with abnormal liver ultrasonography. All patients underwent treatment with doxycycline and aminoglycoside for seven days and doxycycline alone for two months. Most of them responded to antibiotic therapy with rapid regression of symptoms. One patient failed to respond to therapy and presented necrotizing orchitis, as well as abscesses, which required orchectomy. Brucellar infections of the genitalia and lower urinary tract have no specific clinical presentation; the usual laboratory examination is not sufficient to diagnose this kind of infection, therefore it could easily be misdiagnosed. An analytical medical history (including overall dietary habits and recent consumption of non-pasteurized dairy products) could indicate Brucelosis as would the persistence of symptoms despite a one-week antibiotic treatment. In general, patients afflicted by brucellar epididymoorchitis respond to Brucellosis antibiotic therapy, except for some rare cases that present necrotizing orchitis and require surgical treatment.
\end{abstract}

Key-Words: Human brucellosis, epididymoorchitis, brucellar infections, occupational exposure.

Human brucellosis is a potentially life-threatening multisystem zoonotic disease of bacterial origin. The incidence of brucellar infections is characterized by considerable geographical variability. While it is uncommon in the US, northern Europe and Eastern Asia, it is common in the Middle East, southern Europe, and South America [1]. The decline in disease incidence that has been registered worldwide is mainly due to compulsory pasteurization of milk and to the control of the disease in dairy cattle, though a possible role of health education in the decline of human brucellosis is not to be excluded [2]. Genitourinary system involvement occurs in $2 \%$ to $20 \%$ of patients with brucellosis and includes prostatitis, epididymoorchitis, cystitis, pyelonephritis, interstitial nephritis, exudative glomerulonephritis and renal abscesses. Due to its non-specific symptoms, brucellar infections of the urinary tract often remain underdiagnosed and underreported [3]. Epididymis seems to be the most often affected organ of the genitalia and lower urinary tract. Epididymitis due to Brucella can cause serious complications, such as necrotizing orchitis, aspermia and oligospermia, if left untreated [4], and therefore it must be considered in the differential diagnosis of acute scrotum problems [5]. To our knowledge, genitourinary complications of brucellosis have rarely been documented in the medical literature, while published articles describing cases of brucellar epididymoorchitis (BEO) are few in number.

Received on 16 August 2008; revised 2 December 2008.

Address for correspondence: Dr. Stamatiou Konstantinos. 2 Salepoula str., 18536 Piraeus, Greece. Phone:+302104526651. Fax:+302104296987. E-mail: stamatiouk@gmail.com.

The Brazilian Journal of Infectious Diseases 2009;13(2):86-89. (C) 2009 by The Brazilian Journal of Infectious Diseases and Contexto Publishing. All rights reserved.

\section{Material and Methods}

According to the records of the General Hospital of Elefsina "Thriassio", between 1987 (when the General Hospital of Elefsina "Thriassio" was founded) and 2007, 594 cases of epididymoorchitis have been hospitalized in our department. Seventeen out of 594 cases (2.86\%) were diagnosed with epididymoorchitis due to Brucella mellitensis and followed up prospectively. The medical records of these patients were reviewed in order to provide a comprehensive description of this entity. In addition, a literature review was performed using the key words "human brucellosis," "brucellar infections," and "brucellar epididymoorchitis," dating from 1980 to 2008, in order to compare our findings with those provided by the literature. To our knowledge, brucellosis is endemic in Greece and despite the reduction in the incidence of animal brucellosis reported the last 10 years, we suspect that the true disease burden is underestimated.

\section{Results}

Patient Demographics

The mean age of patients with BEO was 49.2, (median 52, range 15-77 years of age). Eleven out of 17 patients were rural residents and four reported occupational exposure (Figure 1). Fifteen patients reported that they might have consumed unpasteurized dairy products, which is a risk factor for brucellosis, 15-20 days before the onset of initial symptoms. Four patients were immigrants (three European and one African).

\section{Signs and Symptoms}

The onset of symptoms was acute ( $<30$ days) in almost all cases (16/17 patients) and sub-acute in one patient. Lower urinary tract symptoms were present in nine patients. Fifteen out of the 17 patients had unilateral involvement, while the 
Figure 1. Brucellar epididymoorchitis cases diagnosed in the General Hospital of Elefsina “Thriassio” between 1987 and 2007.

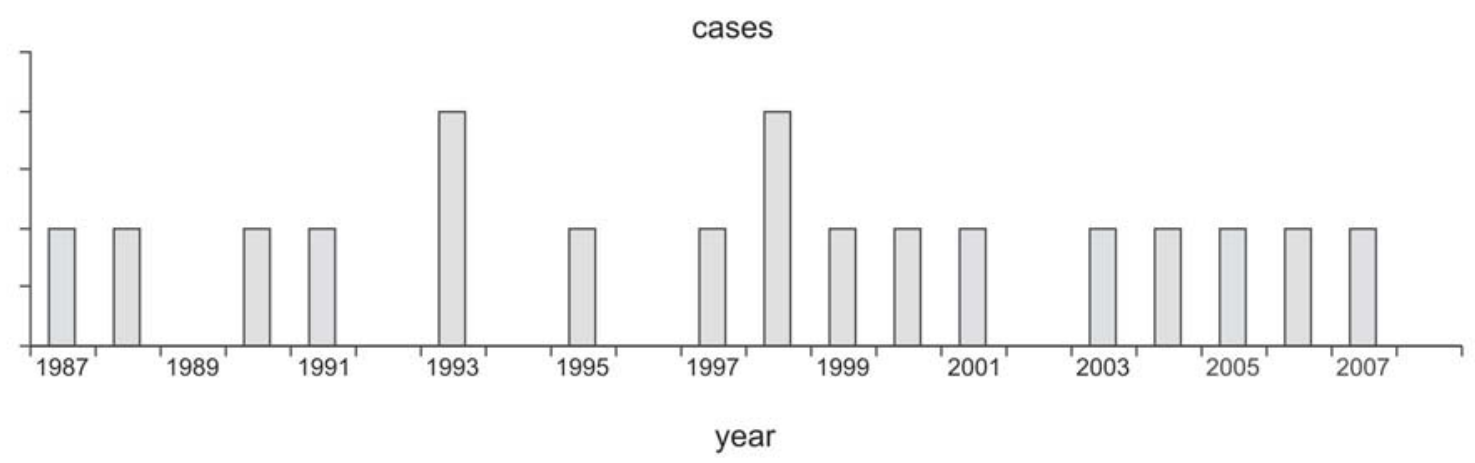

remaining two had bilateral BEO. In one of them, epidedimal swelling occurred bilaterally, almost simultaneously. Symptoms onset was acute, with scrotal pain, epididimal swelling and fever being the most common symptoms reported by the patients. Eight out of the 17 patients were referred to our department because of persistent fever and scrotal swelling; six patients were referred to our department because of persistent scrotal swelling and pain, while the remaining patients were referred to our department because of painless scrotal swelling. Five patients presented with signs and symptoms of systematic disease. All but three of the 17 were diagnosed with non-specific epididymitis and had been treated 1-18 months before the final diagnosis of BEO. None of them responded to the previously-administered one-week antibiotic treatment. The time from onset of symptoms to diagnosis of BEO ranged from 15 days to 1.5 years.

\section{Laboratory Data}

Leukocytosis $\left(>10,500 \mathrm{WBCs} / \mathrm{mm}^{3}\right)$ was found in five of the 17 patients, leukopenia $\left(<4,500 \mathrm{WBCs} / \mathrm{mm}^{3}\right)$ was found in three patients, while the remaining patients had normal white blood cell counts. Anemia (hemoglobin concentration $<13.5$ $\mathrm{g} / \mathrm{dL}$ ) was found in three patients and thrombocytopenia $\left(<150,000\right.$ platelets $/ \mathrm{mm}^{3}$ ) was discovered in two patients. Erythrocyte sedimentation rates (ESRs) were abnormal in 14 out of the 17 patients. Just over half of the patients had a slight to moderate increase in serum hepatic transaminase levels (9/17 patients), most without elevated serum g-glutamyl transpeptidase concentrations. Urinalysis was normal in nine patients, three patients had pyuria and a positive urine culture for Escherichia coli, while the remaining (5/17 patients) had pyuria and a negative urine culture. Since brucellar infections of the genitalia were not suspected initially, specific Brucella urine cultures were not taken. The Wright test was performed on 13 patients and was positive for antibodies to Brucella (titer, >1:160) in all cases. Cultures of blood specimens were made for six patients with febrile epididymoorchitis; all were positive for Brucella spp., while $\beta$-hcg and $\alpha$-ftp values were within normal limits in all three patients with painless scrotal swelling. Epididymal aspiration was performed on one patient and Brucella sp. was isolated.

\section{Ultrasonographic Findings}

Abdominal ultrasonography was performed only on patients who had altered serum hepatic transaminase levels; however, among the nine patients with altered serum hepatic transaminase levels, only two were found to have abnormal liver ultrasonography. Scrotum ultrasonography was routinely performed on all patients. Six out of the 17 patients were found have only unilateral dilation of the epididymis, The testes were enlarged in five patients, four presented hydrocele and two had bilateral epididymoorchitis. Changes in the echotexture of the testes were detected in seven sonograms.

\section{Outcome}

All patients underwent treatment with doxycycline and aminoglycoside for seven days and doxycycline alone for two months. Most of them responded to the antibiotic therapy with rapid regression of their symptoms. One patient failed to respond to therapy and presented necrotizing orchitis, as well as abscesses, which required orchectomy. Three patients experienced relapse.

\section{Discussion}

Brucellosis is endemic in our country, with Brucella melitensis being the most common pathogen [6]. Many efforts have been made by the Ministries of Agriculture and Public Health, in cooperation with the European Union, during the last 10 years in order to reduce the incidence of animal brucellosis, and a large-scale program of animal vaccination was started. Finally, after 2000, a remarkable decrease in the number of reported cases was observed (2.2 per 100,000 in 2003 versus from 1.1 to 5.0 per 100,000 in 1993) [7]. Since there is a very close positive correlation between the incidence of brucellosis in animals and its incidence in humans [8], we assumed that animal vaccination would considerably influence human incidence of this disease and a decrease in the incidence of human brucellosis would be expected. Nevertheless, based on the records of the General Hospital of Elefsina "Thriassio", the number of BEO cases remained steady between 1987 and 2007. We think that it is likely that the true disease burden has been underestimated. In fact, 
recent studies reported increased incidence of animal brucellosis in certain rural areas in Greece, which could be due to illegal importation of infected animals [9-11].

BEO has been reported in up to $20 \%$ of patients with brucellosis. Similar to systematic disease, BEO is rare in the western word but is widespread in the Middle East [12,13]. Its true incidence worldwide is unknown. However, we found 17 out of 594 cases of epididymo-orchitis (2.86\%, this study) to be caused by Brucella mellitensis. This percentage should be interpreted with caution, however, since many other cases of epididymo-orchitis have been diagnosed in the outpatient department. Since they were not hospitalized and therefore not promptly investigated, they might have been misdiagnosed as non-specific epididymo-orchitis. Both clinical and radiological characteristics of BEO have been previously reported $[14,15]$. Seasonal pattern, gradual onset, long duration, typical undulatory fever, absence of serious leukocytosis and lower urinary tract symptoms, and relatively minimal local signs of florid inflammation poses a clinical suspicion of BEO, while heterogenicity, focal echogenicity differences and hydrocele with granularity and/or septation on scrotal ultrasound raise the possibility of BEO rather than non-specific epididymo-orchitis. Only a few BEO series have been reported in sufficient detail to allow analysis and therefore to establish distinct diagnostic criteria [15-20]. Moreover, the above-mentioned characteristics are not always present in patients with BEO and therefore differential diagnosis between $\mathrm{BEO}$ and non-specific epididymo-orchitis is often difficult. Notably, brucellosis is a multi-system disease, with a broad spectrum of non-specific symptoms that generally occur between two weeks and three months after infection, and thus it could be easily misdiagnosed.

In fact, in our study, 14 of the 17 patients had been previously misdiagnosed with non-specific epididymitis. Onset of symptoms was acute in almost all cases, and lower urinary tract symptoms were present in most of patients, as was also found by Khan et al. and Akinci et al. [4,20]. In addition, no seasonal pattern was noticed, while we found leukocytosis in 5/17 patients, different from what was reported by Crosby et al. [21]. In our study, only 7/17 patients presented scrotal ultrasonographic findings that could attributed to BEO, while Ozturk et al. reported as high as $82 \%$ (14). We think that ultrasonography is more useful in enabling exclusion of the possibility of abscesses or tumors than it is in helping to establish the primary clinical diagnosis.

The demographic profile of the patients in this study was similar to those of groups of patients with BEO described elsewhere [15-20], however, the generally low median age of patients presenting BEO needs to be investigated. Although the impact of Brucella infection on the sexual functioning of our patients was not investigated, the relationship between brucellar epididymoorchitis and infertility reported in the study of Akinci et al. [4] indicates that it should also be examined.

Studies of risk factor have concluded that several behavioral factors (dairy products consumption and animal delivery practices) play a very important role in the spread of the disease [8]. Although, in our study, the exact mechanism by which the disease had been transmitted to humans was not clarified, it is suspected that in most of the cases it developed as a result of consuming un-pasteurized, contaminated goat's milk and derivatives that had been infected with B. melitensis. In fact, 15/17 patients reported that they might have consumed un-pasteurized dairy products, while only four reported occupational exposure. Notably, the city of Elefsina is in close proximity with the mountainous rural area of Pili. Most of the residents of this rural area are cattle breeders, and they produce dairy products in small family settings.

The duration of antibiotic therapy for BEO varies considerably among studies; however, it seems that oral doxycycline for two months is as effective as combinationational therapy with doxycycline plus rifampicin, or doxycycline plus streptomycin or rifampicin plus streptomycin. In fact, the relapse rate in our series (3/17) is comparable to that of other studies (11\%-25\%) [22,23] .

\section{Conclusions}

Brucellar infections of the genitalia and lower urinary tract have no specific clinical presentation, while the usual laboratory examination is insufficient to diagnose this kind of infection; therefore, it could easily be misdiagnosed. An analytical medical history (including overall dietary habits and recent consumption of non-pasteurized dairy products) could pose a suspicion of Brucelosis, as would the persistence of symptoms. However, the final diagnosis should be confirmed with laboratory examinations. In general, patients afflicted by Brucellar epididymoorchitis usually have a favorable outcome with standard treatment of brucellosis, except for some rare cases that present necrotizing orchitis and require surgical treatment. Brucellar epididymo-orchitis should be a consideration in the differential diagnosis of patients presenting with signs and symptoms of this entity in areas endemic for brucellosis.

\section{References}

1. Memish Z.A., Balkhy H.H. Brucellosis and international travel. J Travel Med 2004;11:49-55.

2. Sauret J., Vilissova N. Human Brucellosis. JABFP 2002;15:401-6.

3. Khan M.S., Humayoon M.S., Al-Manee M.S. Epididymo-orchitis and brucellosis. Br J Urol 1989;63:87-9.

4 Akinci E., Bodur H., Cevik M.A., et al. A complication of brucellosis: epididymoorchitis. Int J Infect Dis 2006;10:171-7.

5. Reisman E.M., Colquitt L.A. 4th, Childers J. Brucella orchitis: a rare cause of testicular enlargement. J Urol 1990;143:821-2.

6. Taleski V., Zerva L., Kantardjiev T., et al. An overview of the epidemiology and epizootology of brucellosis in selected countries of Central and Southeast Europe. Vet Microbiol 2002;90:147-55.

7. KEEL - Hellenic Center for Infectious Diseases (2004) Epidemiological data. Available at: http://www.keel.org.gr/ stanalysis.

8. Refai M. Incidence and control of brucellosis in the Near East region. Vet Microbiol 2002;90:81-110. 
9. Bikas C., Jelastopulu E., Leotsinidis M., Kondakis X. Epidemiology of human brucellosis in a rural area of NorthWestern Peloponnese in Greece. Eur J Epidemiol 2003;18:267-74.

10. Hadjichristodoulou C., Soteriades E., Goutsianna G. Surveillance of brucellosis in a rural area of Greece: Application of the Computerized Mapping Programme. Eur J Epidemiol 1999;15:277-83.

11. Avdikou I., Maipa V., Alamanos Y. Epidemiology of human brucellosis in a defined area of North Western Greece. Epidemiol Infect 2005;133:905-10.

12. Yetkin M.A., Erdinc F.S., Bulut C., Tulek N. Epididymoorchitis due to brucellosis in central Anatolia, Turkey. Urol Int 2005; 75:235-8.

13. Alballa S.R. Epidemiology of human brucellosis in southern Saudi Arabia. J Trop Med Hyg 1995;98:185-9.

14. Ozturk A., Ozturk E., Zeyrek F., et al. Comparison of brucella and non-specific epididymorchitis: gray scale and color Doppler ultrasonographic features. Eur J Radiol 2005;56:256-62.
15. Papatsoris A.G., Mpadra F.A., Karamouzis M.V., Frangides C.Y. Endemic brucellar epididymo-orchitis: a 10-year experience. Int J Infect Dis 2002;6:309-13.

16 Ibrahim A.I.A., Awad R., Shetty D., et al. Genito-urinary complications of brucellosis. Br J Urol 1988;61:294-8.

17. Guinda-Sevillano C., Arevalo-Velasco J.M., Perez Arbej J.A. Orquitis brucelar. Aportacio'n de una serie de 16 casos. Acta Urol Esp 1995;19:455-8.

18. Arrura A., Pertusa C., Zabala J.A. Brucelosis genital. Arch Esp Urol 1990;43:673-4.

19. Yurdakul T., Sert U., Acar A. Epididymo-orchitis as a complication of brucellosis. Urol Int 1995;55:141-2.

20. Khan M.S., Humayoon M.S., Al-Manee M.S. Epididymo-orchitis and brucellosis. Br J Urol 1989;63:87-9.

21. Crosby E., Llosa L., Miro Quesada M., et al. Hematologic changes in brucellosis. J Infect Dis 1984;150:419-24.

22. Fallatah S.M., Oduloju A.J., Al-Dusari S.N., Fakunle Y.M. Human brucellosis in Northern Saudi Arabia. Saudi Med J 2005;26:1562-6.

23. Navarro-Martýnez A., Solera J., Corredoira J., et al. Epididymoorchitis due to Brucella mellitensis: A retrospective study of 59 patients. Clin Inf Dis 2001;33:2017-22. 